\title{
INFORMES*
}

\section{El Decreto 168/2007, de 12 de Junio, por el que se regula el procedimiento para el reconocimiento de la situación de dependencia y del derecho a las prestaciones del sistema para la autonomía y atención a la dependencia, así como los órganos competentes para su valoración $* *$}

I. El Decreto 168/2007, de 12 de junio, regula el procedimiento para el reconocimiento de la situación de dependencia y del derecho a las prestaciones del Sistema para la Autonomía y Atención a la Dependencia, así como los órganos competentes para su valoración. Trae causa, por tanto, de la Ley estatal 39/2006, de 14 de diciembre, de Promoción de la Autonomía Personal y Atención a las personas en situación de dependencia.

La Ley citada tiene por objeto regular las condiciones básicas que garanticen la igualdad en el ejercicio del derecho subjetivo de ciudadanía a la promoción de la autonomía personal y atención a las personas en situación de dependencia (art. 1.1), definida ésta como "el estado de carácter permanente en que se encuentran las personas que, por razones derivadas de la edad, la enfermedad o la discapacidad, y ligadas a la falta o a la pérdida de autonomía física, mental, intelectual o sensorial, precisan de la atención de otra u otras personas o ayudas importantes para realizar actividades básicas de la vida diaria o, en el caso de las personas con discapacidad intelectual o enfermedad mental, de otros apoyos para su autonomía personal" (art. 2.2).

\footnotetext{
* Sección redactada bajo la dirección de José Ignacio MORILLO-VELARDE PÉREZ.

** Realizado por María del Carmen NÚÑEZ LOZANO, profesora titular de Derecho Administrativo de la Universidad de Huelva.
} 
Para ello, crea el Sistema para la Autonomía y Atención a la Dependencia con la colaboración y participación de todas las Administraciones Públicas (art. 1.1), que en particular pretende responder a una acción coordinada y cooperativa de la Administración General del Estado y las Comunidades Autónoma (art. 1.2) y que no es sino reflejo de la complejidad que presenta en este ámbito la articulación de competencias entre el Estado y las CC.AA ${ }^{1}$. $\mathrm{Si}$ bien el protagonismo corresponde a estas instancias territoriales, debe advertirse que se prevé también la participación de las Entidades Locales en la gestión de los servicios de atención a las personas en situación de dependencia, de acuerdo con la normativa de sus respectivas CC.AA y dentro de las competencias que la legislación vigente les atribuye (art. 12.1).

La Ley protege la situación de dependencia distinguiendo los siguientes tres niveles que regula el art. 7: 1) el nivel de protección mínimo establecido por la Administración General del Estado, que financia el Estado (arts. 9 y 32.1); 2) el nivel de protección que se acuerde entre la Administración General del Estado y la Administración de cada una de las Comunidades Autónomas, a través de convenios que desarrollen el marco de cooperación interadministrativa que se acuerde en el seno del Consejo Territorial del Sistema para la Autonomía y Atención a la Dependencia (art. 10), nivel éste que financian conjuntamente la Administración del Estado y la Comunidad Autónoma (art. 32.3); y 3) el nivel adicional de protección que pueda establecer cada Comunidad Autónoma, que lógicamente financia ella, correspondiéndole también la adopción de las normas de acceso y disfrute que consideren más adecuadas (art. 11.2).

En todo caso, corresponde a las Comunidades Autónomas planificar, ordenar, coordinar y dirigir, en el ámbito de su territorio, los servicios de promoción de la autonomía personal y de atención a las personas en situación de dependencia, así como gestionar en su ámbito territorial los servicios y recursos necesarios para la valoración y atención de la dependencia [art. 11.1.a) y b) ]².

\footnotetext{
${ }^{1}$ Expresivas de ello son las finalidades que la Ley asigna al Sistema: 1) garantizar las condiciones básicas y el contenido común a que se refiere la Ley; 2) servir de cauce para la colaboración y participación de las Administraciones Públicas en el ejercicio de sus respectivas competencias, en materia de promoción de la autonomía personal y la atención y protección a las personas en situación de dependencia; 3) optimizar los recursos públicos y privados disponibles; y 4) contribuir a la mejora de las condiciones de vida de los ciudadanos (art. 6.1).

${ }^{2}$ Se concreta en la Ley que los servicios se han de prestar a través de la oferta pública de la Red de Servicios Sociales por las respectivas CC.AA. mediante centros y servicios públicos o privados concertados debidamente acreditados (arts. 14.2 y 16.1).
} 
El instrumento de cooperación para la articulación del Sistema que prevé la Ley es el Consejo Territorial del Sistema para la Autonomía y Atención a la Dependencia, en cuya composición tienen mayoría las Comunidades Autónomas (art. 8.1) y que ostenta competencias verdaderamente trascendentales en orden a la configuración y funcionamiento del propio Sistema, así como en lo que concierne al alcance y efectividad de los derechos de las personas en situación de dependencia (art.8.2). De entre ellas ha de destacarse la de "acordar el baremo a que se refiere el artículo 27, con los criterios básicos del procedimiento de valoración y de las características de los órganos de valoración" (art. 8.2.e) $)^{3}$.

Esta última referencia nos permite enlazar con el objeto del Decreto 168/2007. Para el acceso a las prestaciones del Sistema es preciso tener reconocida la situación de dependencia ${ }^{4}$, lo que tiene lugar mediante la aplicación de un baremo acordado, como se acaba de mencionar, en el Consejo Territorial y aprobado por Real Decreto ${ }^{5}$. La aplicación la llevan a cabo los

\footnotetext{
${ }^{3}$ Reproducimos a continuación el resto en la medida en que revelan la consideración del Consejo como pieza clave del Sistema: a) acordar el marco de cooperación interadministrativo para el desarrollo de la Ley previsto en el art. 10; b) establecer los criterios para determinar la intensidad de protección de los servicios previstos de acuerdo con los artículos 10.3 y 15; c) acordar las condiciones y cuantías de las prestaciones económicas previstas en el art. 20 y en la disposición adicional primera; d) adoptar los criterios de participación del beneficiario en el coste de los servicios; f) acordar, en su caso, planes, proyectos y programas conjuntos; g) adoptar criterios comunes de actuación y de evaluación del Sistema; h) facilitar la puesta a disposición de documentos, datos y estadísticas comunes; i) establecer los mecanismos de coordinación para el caso de las personas desplazadas en situación de dependencia; j) informar la normativa estatal de desarrollo en materia de dependencia y en especial las normas previstas en el art. 9.1; k) servir de cauce de cooperación, comunicación e información entre las Administraciones Públicas.

${ }^{4}$ La Ley contempla tres grados de dependencia (moderada, severa y gran dependencia), clasificados cada uno de ellos en dos niveles (art. 26). Para los menores de tres años se prevé una escala de valoración específica (DA 13르).

${ }^{5}$ Mediante acuerdo de 23 de marzo de 1007, el Consejo Territorial acordó el baremo, aprobado luego por RD 504/2007, de 20 de abril. Según prescribe el art. 27, el baremo establece los criterios objetivos de valoración del grado de autonomía de la persona, de su capacidad para realizar las distintas actividades de la vida diaria, los intervalos de puntuación para cada uno de los grados y niveles de dependencia, y el protocolo con los procedimientos y técnicas a seguir para la valoración de las aptitudes observadas, en su caso (apartado 3); asimismo valora la capacidad de la persona para llevar a cabo por sí misma las actividades básicas de la vida diaria, así como la necesidad de apoyo y supervisión para su realización por personas con discapacidad intelectual o con enfermedad mental (apartado 4).
} 
órganos de valoración públicos que determina la Comunidad Autónoma conforme a los criterios comunes de composición y actuación que acuerda el Consejo Territorial (art. 27.1), en el seno del denominado "procedimiento para el reconocimiento de la situación de dependencia y del derecho a las prestaciones del Sistema”, cuyos criterios básicos acuerda, como sabemos, el Consejo Territorial (art. 28.5) ${ }^{6}$.

Acordados unos y otros criterios por el Consejo el 22 de enero de $2007^{7}$, la Comunidad Autónoma ha procedido a regular el procedimiento y los órganos competentes del modo que a continuación se expone.

II. En primer término, el Decreto 168/2007 desconcentra en las Delegaciones Provinciales de la Consejería competente en materia de servicios sociales la competencia para valorar la situación de dependencia, resolver los procedimientos para el reconocimiento de la situación de dependencia, para prescribir los servicios y prestaciones y para gestionar las prestaciones económicas que establece la Ley 39/2006 (art. 4.1). Contempla también la participación de las Entidades Locales en el procedimiento para el reconocimiento de la situación de dependencia y, en su caso, del derecho a las prestaciones del Sistema (art. 4.2). Y señala que corresponderá a los Servicios Sociales Comunitarios correspondientes al municipio de residencia de las personas solicitantes iniciar la tramitación del procedimiento, así como la elaboración de la propuesta de Programa Individual de Atención (art. 4.3) ${ }^{8}$.

III. La determinación del grado y nivel de dependencia la llevan a cabo los órganos de valoración, compuestos por el personal definido en la correspondiente relación de puestos de trabajo, pertenecientes a las áreas social o sanitaria e integrado en la estructura administrativa de la Delegación Provincial correspondiente (art. 7.1). Cada Delegación cuenta con un Servicio de Valoración que se constituye como órgano de valoración (art. 7.2).

\footnotetext{
${ }^{6}$ Que reitera también que el Consejo acuerda las características comunes del órgano y profesionales que procedan al reconocimiento.

${ }^{7}$ BOE 7 de junio de 2007.

${ }^{8}$ Recordamos que en los municipios de hasta 20.000 habitantes la coordinación y gestión de los Centros de Servicios Sociales Comunitarios corresponde a las Diputaciones, por delegación de la Junta de Andalucía; y que en los municipios de más de 20.000 habitantes ejercen la competencia de gestión de estos centros, también por delegación de la Junta de Andalucía, los Ayuntamientos [arts. 18.2.b) y 19.2.a) de la Ley 2/1988, de 4 de abril, de Servicios Sociales de Andalucía].
} 
Sus funciones son las siguientes: a) Recabar el informe sobre la salud de la persona solicitante conforme al modelo que se establezca; b) Aplicar el baremo de valoración de los grados y niveles de dependencia y la escala de valoración específica para los menores de tres años; c) Establecer la correspondencia entre el resultado del baremo ( sic) con la información relativa a las condiciones de salud; d) Formular ante la persona titular de la Delegación provincial la propuesta de resolución sobre el grado y nivel de dependencia; e) Prestar asistencia técnica y asesoramiento en los procedimientos contenciosos en que sea parte la Administración de la Junta de Andalucía, en materia de valoración de la situación de dependencia y de su grado y nivel; f) Aquellas otras funciones que le sean legal o reglamentariamente atribuidas (art. 7.3).

Para el ejercicio de sus funciones pueden contar con el asesoramiento de profesionales del Sistema Sanitario Público de Andalucía, con el fin de prestar asistencia técnica en el ámbito de sus competencia (art. 7.4).

IV. El procedimiento para el reconocimiento de la situación de dependencia y del derecho a las prestaciones del Sistema se inicia a instancia de los andaluces y andaluzas residentes en Andalucía que pudieran ser titulares de los derechos establecidos en la Ley $39 / 2006^{9}$ o de quienes ostenten su representación (arts. 2 y 8.1) ${ }^{10}$. Ha de aclararse que el Decreto amplía el ámbito de cobertura que contempla la Ley ${ }^{11}$, pues al amparo de lo establecido en el art.

${ }^{9}$ El Decreto menciona "las personas titulares de derechos", pero obviamente la condición de ser titular del derecho dependerá de la resolución que se adopte al término del procedimiento; de ahí que se exprese con mayor corrección el art. 28.1 de la Ley 39/2006 al señalar que "el procedimiento se iniciará a instancia de la persona que pueda estar afectada por algún grado de dependencia”.

${ }^{10}$ La Junta de Andalucía admite, con buen criterio, que sea el guardador de hecho quien signe la solicitud (vid. http://www.juntadeandalucia.es/igualdadybienestarsocial/export/Personas_Mayores/HTML/Ficheros/sol_depen_homologada.pdf )

${ }^{11}$ Los titulares de derechos, según la Ley, son los españoles que cumplan los siguientes requisitos: a) encontrarse en la situación de dependencia en alguno de los grados establecidos; b) en el caso de los menores de tres años, encontrarse en la situación de dependencia conforme a la escala de valoración específica; c) residir en territorio español y haberlo hecho durante cinco años, de los cuales dos deberán ser inmediatamente anteriores a la fecha de presentación de la solicitud; para los menores de cinco años, el periodo de residencia se exige a quienes ejerzan su guardia y custodia (art. 5.1 y disposición adicional $13^{a}$ de la Ley 39/2006). Se contemplan previsiones específicas para los carentes de nacionalidad española, los españoles no residentes y los emigrantes retornados (art. 5.2, 3 y 4 de la Ley). 
48 de la Ley 8/2006, de 24 de octubre, del Estatuto de Andaluces en el mundo, exime a los emigrantes del cumplimiento del requisito de haber residido en territorio español durante cinco años, de ellos dos inmediatamente anteriores a la fecha de presentación de la solicitud (Disposición Adicional Primera) ${ }^{12}$.

La solicitud, formulada según modelo que ha de aprobar la Consejería, puede presentarse en los Servicios Sociales Comunitarios correspondientes al municipio de residencia del solicitante ${ }^{13}$ (art. 10). Está prevista, no obstante, la utilización de medios telemáticos, informáticos y electrónicos y la aplicación del Decreto 183/2003, de 24 de junio, por el que se regula la información y atención al ciudadano y la tramitación de procedimientos administrativos por medios electrónicos (Internet), aunque se difiere a una Orden de la Consejería el establecimiento de la tramitación telemática de los procedimientos que regula el Decreto (art. 5).

A la solicitud se ha de acompañar la siguiente documentación: a) Documento nacional de identidad de la persona solicitante o, en su defecto, documento acreditativo de su personalidad; b) En su caso, documento nacional de identidad o, en su defecto, documento acreditativo de la personalidad de quien ostente la representación, resolución judicial de incapacitación y documento acreditativo de la representación; c) Certificado de empadronamiento acreditativo de la residencia de la persona solicitante (art. 9).

Una vez presentada, o en su caso completada la solicitud (art. 11), los Servicios Sociales Comunitarios la remiten al órgano de valoración, que debe comunicar a la persona solicitante el día y hora en que los profesionales de tal órgano acudirán al domicilio o lugar de residencia para efectuar la valoración (art. 12.1); sólo excepcionalmente se podrá llevar a cabo la valoración en unas instalaciones diferentes al domicilio de la persona solicitante (art. 12.2). Se trata de una previsión sumamente acertada que tiene en cuenta, no sólo las muy posibles dificultades de desplazamiento de las personas cuyo grado de dependencia se ha de valorar y las molestias de todo tipo que les puede ocasionar el trámite, sino también la mayor fiabilidad de la valoración realizada en el entorno cotidiano de la persona.

\footnotetext{
${ }^{12}$ Aunque ha de notarse que la financiación del primer nivel de dependencia corresponde al Estado.

13 Sin perjuicio de lo dispuesto en el art. 38.4 de la Ley 30/1992.
} 
La valoración se realiza teniendo en cuenta el resultado de los reconocimientos y pruebas pertinentes para la aplicación del baremo, el informe sobre la salud de la persona, el informe sobre el entorno en que viva y, en su caso, las ayudas técnicas, órtesis y prótesis que le hayan sido prescritas (art. 27.5 de la Ley 39/2006 y art. 13.1 y 2 del Decreto que comentamos). Añade el art. 13.3 del Decreto que excepcionalmente el órgano de valoración podrá solicitar los informes complementarios o aclaratorios que considere convenientes, así como recabar de los Servicios Sociales Comunitarios o de otros organismos, los informes médicos, psicológicos o sociales pertinentes cuando el contenido de los antecedentes obrantes en el expediente o las especiales circunstancias de la persona interesada así lo aconsejen ${ }^{14}$.

En este momento, debemos detenernos para consignar una breve reflexión que suscita tanto el art. 27.5 de la Ley como el art. 13.2 del Decreto: si la dependencia es un estado en que se encuentra una persona por razones derivadas de la edad, la enfermedad o la discapacidad, en el que se precisa de la atención de otras personas o de ayudas importantes o de apoyos, pero en el que no influyen estas ayudas o apoyos que la persona pueda venir recibiendo de su entorno (art. 2.2 de la Ley), no parece razonable que para el reconocimiento de la situación de dependencia se pondere un informe sobre el entorno en que vive la persona, pues ésta se encontrará o no en la situación de dependencia que describe la Ley y que el baremo detalla, tanto si circunstancialmente se encuentra asistida como si no lo está ${ }^{15}$.

Tras la valoración, los órganos encargados de este cometido deben elevar a la persona titular de la Delegación provincial la propuesta de resolución, con el siguiente contenido: 1) dictamen sobre el grado y nivel de dependencia; 2) especificación de los servicios y prestaciones que la persona puede requerir; 3)

${ }^{14}$ No se aplica lo dispuesto en estos arts. 12 y 13 a las personas que tengan reconocido el complemento de la necesidad del concurso de otra persona según el baremo del Anexo " del RD 1971/1999, de 23 de diciembre, de procedimiento para el reconocimiento, declaración y calificación del grado de minusvalía, pues a ellos se les reconoce el grado y nivel que les corresponda, en función de la puntuación específica otorgada por el citado baremo, de acuerdo con la tabla establecida en la disposición adicional primera 2 del RD 504/2007, de 20 de abril (Disposición Adicional segunda del Decreto).

${ }^{15}$ De hecho, entre los criterios de aplicación del Anexo I del RD 504/2007, de 24 de abril, se encuentra el siguiente: "se valora la necesidad de apoyo de otra persona en la actividad o tarea aunque la persona valorada lo esté recibiendo actualmente y con independencia de éste" (la cursiva es nuestra). 
en su caso, el plazo máximo en que deba efectuarse la primera revisión del grado y nivel dictaminados (art. 14).

La resolución del procedimiento corresponde a la persona titular de la Delegación Provincial y en ella se han de determinar los mismos extremos que contiene el dictamen, con la salvedad de que se ha de indicar la efectividad del derecho a la prestaciones de dependencia conforme al calendario establecido en la Disposición Final Primera de la Ley 39/2006 [art. 15.1.a) del Decreto]. Ello es así porque la efectividad del derecho es progresiva y gradual: alcanza en el primer año a quienes sean valorados en el Grado III de Gran Dependencia, niveles 2 y 1 ; en el segundo y tercer año a quienes sean valorados en el Grado II de Dependencia Severa, nivel 2; en el tercero y cuarto año a quienes sean valorados en el Grado II de la Dependencia Severa, nivel 1; en el quinto y sexto año a quienes sean valorados en el Grado I de Dependencia Moderada, nivel 2; en el séptimo y octavo año, a quienes sean valorados en el Grado I de Dependencia Moderada, nivel I.

El plazo para resolver y notificar es de tres meses, computado a partir de la fecha de entrada de la solicitud en el registro de los Servicios Sociales Comunitarios del municipio de residencia de la persona solicitante; el silencio tiene carácter negativo (art. 15.2 del Decreto). Ha de tenerse en cuenta que se produce la caducidad del procedimiento ex art. 92 de la Ley 30/1992 cuando la valoración resulte imposible por causas imputables a la persona solicitante (art. 12.3 del Decreto).

La resolución de reconocimiento de la situación de dependencia tiene validez en todo el territorio del Estado; ha de comunicarse a los Servicios Sociales Comunitarios correspondientes al municipio de residencia de la persona interesada; su eficacia queda demorada hasta la aprobación del correspondiente Programa Individual de Atención, que más adelante examinaremos con mayor detalle; y es recurrible en alzada ante la persona titular de la Consejería (art. 15.3 y 4 del Decreto).

Por último, se regula también sucintamente el procedimiento para revisar el grado o nivel de dependencia. Ésta, según hemos visto, se define como una situación de carácter permanente, en el sentido de continua o prolongada en el tiempo, lo que no quiere decir que sea perpetua ni invariable; por eso, los arts. 30 de la Ley 39/2006 y 16 del Decreto prevén que pueda producirse una mejoría o un empeoramiento de la situación de dependencia, en cuyo caso se ha iniciar el procedimiento de revisión, a instancia de la persona beneficiaria, de sus representantes o de oficio por la Delegación Provincial. El mismo 
procedimiento se sigue cuando concurre un error de diagnóstico o en la aplicación del correspondiente baremo, aunque obviamente se trata de figuras distintas: el primer caso (mejoría o empeoramiento de la situación) supone la revocación del acto válido, mientras que el segundo (error) es un supuesto de revisión de un acto inválido, cuya modificación o declaración de invalidez debiera producirse mediante el cauce del recurso de alzada, mediante el procedimiento de declaración de nulidad (art. 102 de la Ley 30/1992) o mediante la declaración de lesividad y ulterior recurso contencioso-administrativo (art. 103 de la Ley 30/1992).

V. Como antes se anticipó, la resolución que pone fin al procedimiento para el reconocimiento de la situación de dependencia no es eficaz hasta que se apruebe el Programa Individual de Atención (PIA), en el que se concreta el alcance del derecho prestacional. Su contenido es el siguiente: a) Datos y circunstancias personales y familiares de la persona en situación de dependencia; b) Servicio o servicios prescritos, con indicación de las condiciones específicas de la prestación de éste, así como de la participación que en el coste del mismo pudiera corresponder a la persona en situación de dependencia según su capacidad económica; c) En su caso, de no ser posible el acceso a un servicio público o concertado de atención y cuidado, prestación económica vinculada al servicio; d) Excepcionalmente, prestación económica para cuidados familiares, cuando la persona beneficiaria esté siendo atendida en su entorno familiar y se reúnan las condiciones adecuadas de habitabilidad de la vivienda, con indicación de las condiciones específicas de acceso a dicha prestación; e) En su caso, prestación económica de asistencia personal, con indicación de las condiciones específicas de acceso a dicha prestación (art. 18.2).

A la vista de este contenido, resulta evidente que la resolución de reconocimiento de la situación de dependencia por sí sola no puede surtir efectos, aunque cabe plantearse por qué razón se exige el PIA como pieza distinta de esa primera resolución de reconocimiento de la situación de dependencia, en la que ya han de determinarse los servicios y prestaciones que, en su caso, corresponden (art. 15.1.b). Bastaría, pues, con integrar el contenido del PIA en la resolución de reconocimiento del derecho, en la parte de su contenido que determina los servicios y prestaciones que corresponden a la persona. Sin embargo, ha de tenerse en cuenta que, como ya se ha explicado, la Ley 39/2006 establece lo que se ha dado en denominar una "calendarización" del acceso a las prestaciones, de modo que cabe que se reconozca la situación de dependencia y los servicios y prestaciones que en su caso corresponderían a la persona interesada pero que, sin embargo, 
éstos no puedan hacerse efectivos aún; de ahí que la aprobación del PIA tenga lugar en un momento posterior ${ }^{16}$. Por otra parte, el reconocimiento de la situación de dependencia tiene validez en todo el territorio del Estado y de ahí que en la misma sólo se llegue a precisar, con carácter abstracto, qué servicios o prestaciones corresponden a la persona, quedando pues demorada la fijación de las condiciones concretas y específicas de la asistencia a la aprobación del PIA.

$\mathrm{Al}$ margen ahora de estas razones que justifican la opción seguida por el legislador, ha de reconocerse que en teoría la previsión de dos resoluciones diferentes pudiera presentar una ventaja añadida en la medida en que en el procedimiento para el reconocimiento de la situación de dependencia no se ha de aportar, de conformidad con lo que dispone el articulado del Decreto, documentación acreditativa de la capacidad económica del solicitante, lo que sí se exige en cambio para la aprobación del PIA, puesto que en éste se establecen las condiciones de participación en el coste del servicio. De ahí que pudiera considerarse una garantía de "imparcialidad" la circunstancia de no conocerse de antemano la capacidad económica, al menos de forma fehaciente, puesto que se impediría que la resolución de reconocimiento de la situación de dependencia pudiera estar contaminada por previsiones anticipadas sobre el coste que entrañara la prestación del servicio para la Administración.

Sin embargo, ya el informe sobre el entorno que se ha de ponderar para el reconocimiento de la situación de dependencia puede empañar esa independencia a que acabamos de aludir. Por otra parte, en el modelo de solicitud para el reconocimiento de la situación de dependencia que se muestra en la página web de la Consejería para la Igualdad y Bienestar Social ${ }^{17}$ se recaban una serie de datos e informaciones que tienen sentido o pueden estar justificados en orden a la elaboración del PIA, pero que en línea de principios no son necesarios para el reconocimiento de la situación de dependencia. Si a ello se añade que la firma y presentación de la solicitud, conforme al modelo reseñado, implica la autorización para el suministro de datos de carácter tributario a los órganos correspondientes de la Consejería, se comprende que la garantía de imparcialidad que pudiera entrañar la diferenciación entre el reconocimiento de la situación de dependencia y el PIA no existe realmente.

\footnotetext{
${ }^{16}$ En este caso, la propuesta de PIA se elabora en los tres meses anteriores al inicio de su año de implantación (art. 18.5).

${ }^{17}$ http://www.juntadeandalucia.es/igualdadybienestarsocial/export/Personas_Mayores/HT ML/Ficheros/sol_depen_homologada.pdf
} 
La propuesta del PIA la elaboran los Servicios Sociales Comunitarios que hayan iniciado la tramitación del procedimiento, una vez que se les haya comunicado la resolución de reconocimiento de la situación de dependencia y siempre que la efectividad del derecho deba producirse en el año en que se haya dictado la misma. A estos efectos deben requerir a la persona beneficiaria para que en el plazo de diez días hábiles presenten la siguiente documentación: a) Declaración de los Impuestos sobre la renta y sobre el patrimonio de las personas Físicas, referidas al período impositivo inmediatamente anterior, con plazo de presentación vencido, a la fecha de efectividad del derecho; si no estuvieren obligados a presentarlas, certificación de retenciones de rendimientos percibidos o, en su defecto, declaración responsable de ingresos y declaración sobre la titularidad de los bienes inmuebles, así como los bienes muebles que reglamentariamente se determinen; b) En su caso, documentación acreditativa de las circunstancias personales y familiares alegadas por la persona interesada (art. 17.1).

En el curso del procedimiento los Servicios Sociales Comunitarios deben realizar un informe social en el que se detalle la situación social, familiar y del entorno de la persona en situación de dependencia (art. 17.3). Y ha de darse también participación a la persona beneficiaria o, en su caso, a su familia o entidades tutelares que la representen (art. 17.4).

Una vez elaborada la propuesta del PIA se remite a la Delegación Provincial, junto con las declaraciones, la documentación acreditativa del trámite de audiencia así como el informe social sobre la situación familiar y del entorno de la persona declarada en situación de dependencia; la remisión debe producirse en el plazo máximo de dos meses desde la recepción de la resolución de reconocimiento del derecho (art. 17.4).

La aprobación corresponde a la persona titular de la Delegación Provincial de la Consejería, previas las comprobaciones que procedan (art. 18.1). La aprobación y notificación debe producirse en el plazo máximo de tres meses desde la fecha de recepción de la resolución de reconocimiento de la situación de dependencia por los Servicios Sociales Comunitarios (art. $18.3)^{18}$.

18 Salvo, como es natural, que el derecho de acceso a los servicios y prestaciones correspondientes deba hacerse efectivo en año distinto a aquel en que se haya dictado la resolución de reconocimiento de la situación de dependencia (art. 18.5) o que se trate de un traslado permanente de domicilio desde otra Comunidad Autónoma a la de Andalucía (art. 18.6). 
Contra la resolución por la que se apruebe el PIA cabe recurso de alzada ante la persona titular de la Consejería (art. 18.7). Y puede ser revisado por la Delegación Provincial: a) a instancia de la persona interesada o de sus representantes legales; b) a instancia de los Servicios Sociales Comunitarios correspondientes; c) de oficio, cuando las circunstancias lo aconsejen y, en todo caso, cada tres años; d) como consecuencia del traslado de residencia a la Comunidad Autónoma de Andalucía (art. 19.1). Para la revisión se sigue, en lo que resulte procedente, las normas establecidas para la aprobación del PIA (art. 19.2).

VI. El Capítulo V del Decreto , bajo la rúbrica "seguimiento y control", vuelve a ocuparse de la revisión. Dispone el artículo 20 que las prestaciones reconocidas pueden ser modificadas o extinguidas en función de la situación personal del beneficiario o beneficiaria: a) cuando se produzca variación de cualquiera de los requisitos establecidos para su reconocimiento; b) por incumplimiento de las obligaciones reguladas en la Ley 39/2006 ${ }^{19}$. El procedimiento se inicia de oficio o a instancia de la persona beneficiaria y en el mismo debe darse audiencia a la persona beneficiaria o a su representante. La resolución la dicta la Delegación Provincial de la Consejería.

La Delegación Provincial es la instancia responsable del seguimiento de la correcta aplicación del PIA; en el supuesto en que las personas vivan en su domicilio, la Delegación Provincial debe contar con la colaboración de los Servicios Sociales Comunitarios correspondientes al municipio de residencia de la persona que se encuentre en situación de dependencia (art. 21). En términos generales, se señala que la Administración de la Junta de Andalucía, a través de sus órganos competentes en cada caso, y los Servicios Sociales Comunitarios velarán por la correcta aplicación o utilización de los fondos públicos, prestaciones, servicios y cuantos beneficios se deriven del Sistema para la Autonomía y Atención de la Dependencia (art. 22).

\footnotetext{
${ }^{19}$ De las obligaciones se ocupa específicamente el art. 4.4 de la Ley: "las personas en situación de dependencia y, en su caso, familiares o quienes les representen, así como los centros de asistencia, estarán obligados a suministrar toda la información y datos que le sean requeridos por las Administraciones competentes, para la valoración de su grado y nivel de dependencia; a comunicar todo tipo de ayudas personalizadas que reciban, y a aplicar las prestaciones económicas a las finalidades para las que fueron otorgadas; o a cualquier otra obligación prevista en la legislación vigente". Del catálogo de infracciones que contiene el art. 43 de la Ley se deducen también obligaciones, amén de que el art. 45 (en cuyo análisis no procede entrar ahora, como tampoco procede respecto del art. 43), sobre las sanciones, también contempla la pérdida de las prestaciones y subvenciones para las personas beneficiarias.
} 
VII. Hasta aquí la exposición de los aspectos más relevantes del Decreto 168/2007, que de suyo y como se reconoce en su Exposición de Motivos "permite la puesta en marcha y el acceso de la ciudadanía andaluza al Sistema para la Autonomía y Atención a la Dependencia”. Queda un largo camino por andar hasta que la Ley 39/2006 alcance su plena aplicación, no exento desde luego de incertidumbres y, por lo mismo, de desconfianza respecto del éxito o del fracaso del Sistema. Esperemos, en cualquier caso, que el llamado "cuarto pilar del sistema de bienestar" alcance a satisfacer las expectativas creadas, sin imponer a las familias la carga del alejamiento de la persona dependiente ${ }^{20}$.

${ }^{20}$ En la medida en que la Ley diseña un sistema en el que la atención por cuidadores no profesionales (la familia) es excepcional (vid. el art. 14). 